\title{
Fault Diagnosis of Analog Circuits based on Wavelet Packets
}

\author{
Yigang He ${ }^{1}$,Yanghong Tan ${ }^{1}$ and Yichuang Sun ${ }^{2}$ \\ 1 College Of Electrical \& Information Engineering, Hunan University, \\ Changsha, 410082, P.R.China \\ 2 Department of Electronic, Communication and Electrical Engineering, University of \\ Hertfordshire, Hatfield AL10 9AB, United Kingdom
}

\begin{abstract}
A fault diagnosis method for analog circuits based on wavelet packets is developed in this paper. The sampled signals from the test nodes are decomposed by wavelet packets and the feature vectors extracted are applied to neural networks for identifying the faults. The proposed method is characterized by minimizing ambiguity groups as well as simplifying neural network architecture, reducing training cost and maximizing the diagnosability. Simulation results of diagnosing a four-op-amp filter circuit have confirmed the validity of the propose technique.
\end{abstract}

\section{Introduction}

There has been a growing interest in the last two decades in the development of automatic tools for testing and diagnosis of electronic circuits and systems. The testing techniques for digital circuits have been mature and cost effective, while the testing of analog and mixed analog-digital systems is more complicated and less understood and yet relevant to applications. In fact, the automatic fault testing and diagnosis for analogue circuits is subject to many 2 difficult factors, such as poor fault models, noise, nonlinearity and tolerance effects $[1,2]$. Some methodologies of general validity have been proposed and developed in recent years [3-9]. Neural networks (NN) have been applied to a variety of problems in the areas of pattern recognition, signal processing, image processing, process identification, etc. The classifiers in terms of neural networks trained on the fault dictionary examples have been successfully applied to fault diagnosis, providing satisfactory results while minimizing computation costs [3-7].

Wavelet-based approaches to detect abrupt faults in dynamic systems by decomposing output signals into elementary building blocks by wavelet transforms have been investigated, where the local changes of the signals caused by the faults can be identified by analyzing the non-stationary components of the output signals. However, little literature has been available in the fault analysis and diagnosis of analog circuits using wavelets. References $[10,11]$ proposed a neural network based fault diagnosis method using

0-7803-8560-8/04/\$20.00@2004IEEE wavelets as a preprocessor to reduce the number of input features to a manageable size. Unfortunately, the decomposition based on wavelet transforms $[10,11]$ of a signal into approximation signals and detail signals is limited to the approximate signals. Additional processors are needed to decompose the details, resulting in the increase of computation cost.

The research presented here is to attempt to exploit wavelet packets to extract appropriate feature vectors from the signals sampled from the circuit under test (CUT) under various faults. The processing contains details as well as approximation coefficients. Optimal sets for training neural networks after normalization and principal component analysis (PCA) are transformed and the output of the neural networks reveals the fault patterns. This is a model-free technique due to the fact that the faults of a circuit are claimed and classified based on the energy of the output signal in various resolutions of its wavelet packet transforms.

\section{WAVELET PACKET-BASED FEATURE EXTRACTION}

\subsection{Wavelet Packets Analysis}

Wavelet packets is a signal-dependent subband decomposition exploiting filter banks with rich orthogonal basis functions suited to the signal properties. A pair of low-pass (LP) and high-pass (HP) filters used to yield two sequences downsampled by a factor of two capture different frequency subband features of the original signal. This forms one resolution of wavelet packet decomposition, which can be iteratively repeated to partition the frequency subband (FS) into narrower bands. Then the filter banks consisting of several band-pass (BP) filters each are tuned to pick up certain frequency information.

In this implementation, the difference of the LP and HP offers a coarse resolution illustrating the basic structure of the FS and fine resolution showing the finer detail of the FS, respectively. At each level, the approximations and details are regrouped into a pyramid structure known as the Laplacian pyramid $[10,11]$. 
Assume $g_{j}^{n}(t) \in U_{j}^{m}$, where the $U_{j}^{m}$ is the closed packet space of function $\mathrm{u}_{\mathrm{n}}(\mathrm{t})$, and let

$$
\begin{aligned}
& g_{j}^{n}(t)=g_{j}^{2 n}(t)+g_{j}^{2 n+1}(t) \\
= & \sum_{l} d_{l}^{j, n} u_{n}\left(2^{j} t-l\right) \\
d_{l}^{j, 2 n} & =\sum_{k} a_{k-2 l} d_{k}^{j+1, n} \\
d_{l}^{j, 2 n+1} & =\sum_{k} b_{k-2 l} d_{k}^{j+1, n}
\end{aligned}
$$

Unlike the wavelet transform [10, 11] which gives octave band decompositions of the frequency axis, the wavelet packet analysis can apply the refiner tree whose structure depends on the signal properties, and among these, the full tree divides the frequency axis into equal-bandwidth components yielding a linear division of the spectrum similar to the short-time Fourier Transform (STFT).

\subsection{Creation of Wavelet Packets}

The technique of computing the wavelet packets for an orthogonal wavelet is not complicated. A pair of filters $h(n)$ and $g(n)$ of length $2 \mathrm{~N}$ corresponding to the given orthonormal wavelet basis are constructed with the ratio $1 / \operatorname{sqrt}(2)$ multiplied to the associated reconstruction LP and HP filters. Then the function sequence $\left[\mathrm{U}_{\mathrm{n}}(\mathrm{x}), \quad(\mathrm{n}=0,1,2 \ldots)\right]$ is defined as $[10,11]$

$$
\begin{aligned}
& u_{2 n}(t)=2 \sum_{k=0}^{2 N-1} h(k) u_{n}(2 t-k) \\
& u_{2 n+1}(t)=2 \sum_{k=0}^{2 N-1} g(k) u_{n}(2 t-k)
\end{aligned}
$$

That is, $U_{j+1}^{n}=U_{j}^{2 n}+U_{j}^{2 n+1} \quad \mathrm{j} \in \mathrm{Z}, \mathrm{n} \in \mathrm{Z}_{+}$

\subsection{De-noising Using Wavelet Packet}

A noisy signal $\mathrm{s}(\mathrm{t})$ can be written as $\mathrm{s}(\mathrm{t})=\mathrm{f}(\mathrm{t})+\sigma \mathrm{e}(\mathrm{t})$, where $f(t)$ and $e(t)$ are the principal content and the noise in a circuit. There are two sources of noise: interior noise, for example, thermal noise of a resistor or scintillation noise generated by a semi-conductor element and exterior noise such as disturbance of input signals. Wavelet packet decomposition being accomplished, detail sections are the superposition of

There should be strong variation in the energy of every frequency band under various faults. So the feature vectors can be created as follows:

Residue signals are obtained by wavelet packet analysis and the decomposed coefficients are obtained under multi-resolution as candidate feature sets. details of $f(t)$ and $e(t)$.

The method described in $[10,11]$ selects the approximation coefficients as features from the output node of the circuit and treating the details as noise and just setting them to zero probably leads to losing valid information resulting in high probability of ambiguity cases. The research presented here is to extract candidate features generated from the test points by wavelet de-noising and wavelet decomposition. Then optimal feature vectors to train neural networks are obtained by PCA and normalization of approximation and detail coefficients.

\subsection{Feature Extraction}

In this paper, the computation of the optimal complete sub-tree of order two with an entropy type criterion is executed based on the tree structure and the data structure, corresponding to a wavelet packet decomposition of the original signal $s(t)$, at level $\mathrm{N}$, with a particular wavelet basis. Let the decomposition coefficient $(i, j)$ in the optimal tree be $S_{i j}$, the original $\mathrm{S}$ can be expressed as $[10,11]$

$$
S=S_{N, 0}+S_{N, 1}+S_{N, 2}+\cdots+S_{N, 2^{N}-1}
$$

That is, $2^{\mathrm{k}}$ subbands are related to $\mathrm{W}_{\mathrm{j}}$ :

$$
W_{j}=U_{j-k}^{2^{k}} \oplus U_{j-k}^{2^{k}-1} \oplus \cdots \oplus U_{j-k}^{1}
$$

So, for an arbitrary wavelet series,

$$
\begin{aligned}
& g_{j}(t)=\sum_{n} d_{j}^{n} \varphi\left(2^{j} t-n\right)=\sum_{n} d_{j}^{n} \mu_{1}\left(2^{j} t-n\right) \\
& g_{j, k, m}(t)=\sum_{n} d_{j}^{j, k, m} \mu_{1}\left(2^{j-k} t-n\right), \\
& m=0,1, \ldots 2^{k-1}
\end{aligned}
$$

Table 1 Frequency ranges

\begin{tabular}{|l|l|l|l|l|}
\hline Signals & $\mathrm{S}_{\mathrm{N}, 0}$ & $\mathrm{~S}_{\mathrm{N}, 1}$ & $\mathrm{~S}_{\mathrm{N}, 2} \quad \ldots$ & $\mathrm{S}_{\mathrm{N}, 2}{ }^{\mathrm{N}}-1$ \\
\hline $\begin{array}{l}\text { Frequency } \\
\text { range }\end{array}$ & {$\left[0, \frac{1}{N}\right]$} & {$\left[\frac{1}{N}, \frac{2}{N}\right]$} & {$\left[\frac{2}{N}, \frac{3}{N}\right]$} & {$\left[\frac{N-1}{N}, 1\right]$} \\
\hline
\end{tabular}

Assuming that the minimal frequency in the $x(t)$ is 0 and the maximal is 1 , then the frequency range occupied by the signals $\mathrm{S}_{\mathrm{N}, \mathrm{j}}\left(\mathrm{j}=0,1,2, \ldots 2^{\mathrm{N}}-1\right)$ can be illustrated as in Table 1.

$\mathrm{T}=\left[\mathrm{T}_{\mathrm{N} 0}, \mathrm{~T}_{\mathrm{N} 1}, \mathrm{~T}_{\mathrm{N} 2}, \ldots, \mathrm{T}_{\mathrm{Nm}}\right.$, $]$, where $\mathrm{T}_{\mathrm{j}}$ is the energy of frequency band $\mathrm{j}$ of the residue signals and $\mathrm{m}$ is the number of sample points, are appropriate features to be extracted.

PCA is applied to reduce the dimensionality of the 
feature vectors and thus reduce the input space of the neural networks employed in the fault classification after wavelet transforms, while preserving relevant information for fault identification and removing redundant and irrelevant information being side effects on the classification performance.

Data normalization $[8,9]$ is adopted to avoid large dynamic ranges resulting from the non-convergence of neural networks, that is $\mathrm{T} / \mathrm{E}=\left[\mathrm{T}_{\mathrm{N} 0}, \mathrm{~T}_{\mathrm{N} 1}, \mathrm{~T}_{\mathrm{N} 2}, \ldots\right.$, $\left.\mathrm{T}_{\mathrm{Nm}},\right] / \mathrm{E}$, where $\mathrm{E}$ is the $1_{2}$-norm of the vector $\mathrm{T}$ or singular patterns which differ from the other pattern orders in their magnitudes.

\subsection{Neural Networks}

Neural networks (NN) have been applied to a variety of problems in the areas of pattern recognition, signal processing, image processing, process identification, etc. The classifiers in terms of neural networks trained on the fault dictionary examples have been successfully applied to fault diagnosis, providing satisfactory results while minimizing computation cost [3-7].

A three-layer back propagation neural network (BPNN) can be used as a classifier for its capability of approximating arbitrary functions [3, 7, 11]. The summed squared error performance function is expected to be minimum by feeding information forward and feeding error backward, thus updating the weights and bias according to its learning algorithms. A momentum and adaptive learning rule is adopted to reduce the sensitivity of the local details of error surfaces and shorten the learning time on speculating the experience.

\subsection{Proposed Algorithm}

The sketch of the proposed algorithm is given in Fig.1. It is a wavelet packet neural network (WPNN) immuning from convergence to its local minimum of multi-layer feedback neural network based on wavelet packets.

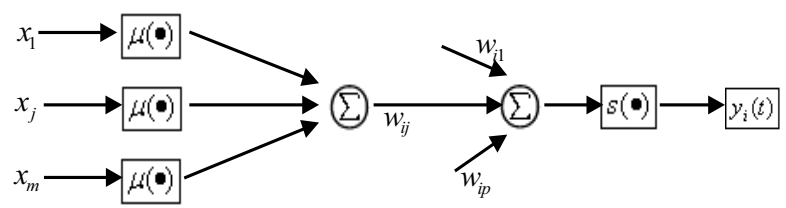

Fig.1 The proposed algorithm

The network function of mapping from $\mathrm{R}^{\mathrm{m}}$ to $\mathrm{R}^{\mathrm{n}}$ can be expressed as

$$
y_{i}(t)=s\left[\sum_{j=1}^{p} w_{i j}\left[\sum_{k=1}^{m} x_{k}(t) \mu(\cdot)\right]\right]
$$

In equation (5), s(.) and $\mu($.$) are the general sigmoid$ nonlinear function and wavelet base, and $\mathrm{x}_{\mathrm{k}}(\mathrm{t})$ and $\mathrm{y}_{\mathrm{i}}(\mathrm{t})$ are the k-th input and i-th output, respectively.

The sum square error performance function [11] is given by

$$
E=\frac{1}{2} \sum_{l=1}^{N} \sum_{i=1}^{q}\left[y_{d, i}^{l}(t)-y_{i}^{l}(t)\right]^{2}
$$

which is expected to be minimum.

The weights in equation (5) are updated by the following [13]

$$
\begin{aligned}
& w_{i j}(k+1)=w_{i j}(k)+\alpha_{w} \cdot D w_{i j}(k)+ \\
& \eta_{w} \cdot\left[w_{i j}(k)-w_{i j}(k-1)\right] \\
& D w_{i j}=-\frac{\partial E}{\partial w_{i j}}
\end{aligned}
$$

where $\alpha_{w}$ is the learning rate for updating weights $\mathrm{w}_{\mathrm{ij}}$ and $\eta_{w}$ the momentum factor, respectively.

From equations (5) and (6) we can derive [12]

$$
\begin{aligned}
& D w_{i j}=\sum_{l=1}^{N} \sum_{i=1}^{q} \sum_{k=1}^{m}\left[y_{d, i}^{l}(t)-y_{i}^{l}(t)\right] . \\
& x_{k}^{l}(t) \mu(\cdot) \frac{\partial}{\partial w_{i j}} S\left(\sum_{j=1}^{p} w_{i j} \sum_{k=1}^{m} x_{k}^{l}(t) \mu(\cdot)\right)
\end{aligned}
$$

The algorithm proposed here contains three main steps:

(1) Extraction of candidate patterns and feature vectors

Candidate features are extracted from the circuit's test points determined by sensitivity analysis and Pspice simulations are applied to obtain typical signals of test points. Then optimal features for training neural networks are obtained by selecting candidate sets from wavelet packet coefficients processed by de-noising, PCA and normalization.

\section{(2) Design and training of neural networks}

A two-layer feedback neural network whose input and output numbers are equal to those of the training patterns and fault classes, respectively is used. The target output of the neural network is a vector $e_{1}$ of zeros except for the 1-th entry, which is equal to 1. The neural network is trained by the optimal features selected in the aforementioned procedure to converge to its global minimum.

(3) Fault diagnosis

Inputting the neural network with the measured data from the CUT due to the change in feature values, the outputs of the WPNN will show the fault patterns.

\section{CIRCUIT EXAMPLE AND DIAGNOSIS RESULTS}

A four op-amp biquad high-pass filter illustrated in 
Fig. 2 with the presence of component tolerances 5\% to $10 \%$ is used in our study. The same fault set as that in $[10,11]$ is used and the diagnosis results are compared with those in $[10,11]$. The impulse responses of the circuit are sampled to form the candidate features with the filter stimulated by a single pulse of height of $5 \mathrm{~V}$ and duration of $10 \mathrm{us}$. To extract the candidates, wavelets packets is constructed for a wavelet 'db3' on dyadic intervals [12]. Then the sampled waveforms are de-noised using the wavelet packets and the optimal sub-tree (shown in Fig.3)

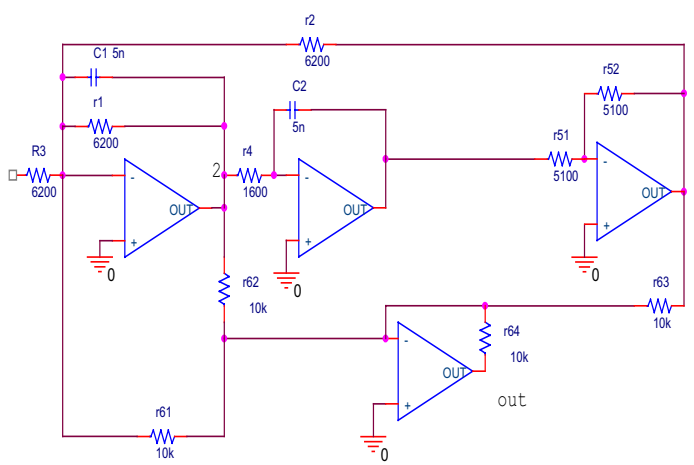

Fig.2 A four op-amp biquad high-pass filter

Using the technique proposed in this paper, we have studied the circuit in Fig.2. As mentioned the measured fault features and the feature vectors by Monte-Carlo PSpice simulations are selected to determine the faults. The algorithm cannot identify all the faults because the features are overlapped to some extent when the component tolerances are near $10 \%$. Nevertheless, the diagnosability achieved is high, about $98.76 \%$. In particular, the study indicates that our system can distinguish the fault classes $\mathrm{C}_{2} \downarrow$ and $\mathrm{R}_{2} \uparrow$ which are misclassified in $[10,11]$. Besides, the fault set between the NF (no fault or fault free) and $R_{2}$ $\uparrow$ that cannot be distinguished in $[10,11]$ have been identified correctly. However, we cannot identify the faults between $\mathrm{C}_{2} \downarrow$ and $\mathrm{R}_{4} \downarrow$ or between $\mathrm{R}_{2} \uparrow$ and $\mathrm{R}_{4} \downarrow$.

\section{CONCLUSIONS}

Candidate features extracted from the energy in every frequency band of the de-noised signals sampled from the test nodes in a circuit are employed to select the optimal feature vectors by PCA, normalization and wavelet multiresolution. Then the optimal feature sets are used to train the wavelet neural networks combining wavelets with neural networks. The proposed method is characterized by its high diagnosability. It can distinguish the ambiguity sets or some misclassified faults that some other methods cannot identify. However, the overlapped ranges appear as the component tolerances increase. Single fault cases considered in this paper, the proposed method is also being generalized for multi-fault diagnosis.

\section{ACKNOWLEDGMENTS}

The authors wish to thank the Natural Science Foundation Council of China under Grant No.50277010, Doctoral Special Fund of Ministry of Education, No.20020532016 and the Fund of Outstanding Young Scientist of Hunan University of matching the de-noised signals is obtained with respect to the "log energy" entropy-type criterion [12]. So the optimal sets are obtained.

We adopt a WPNN architecture of $\mathrm{N}_{1}-42-\mathrm{N}_{2}$ with a view to identifying the fault patterns, where $\mathrm{N}_{1}$ is the number of input patterns and $\mathrm{N}_{2}$ the number of fault patterns. The responses of the neural network declare the faults.

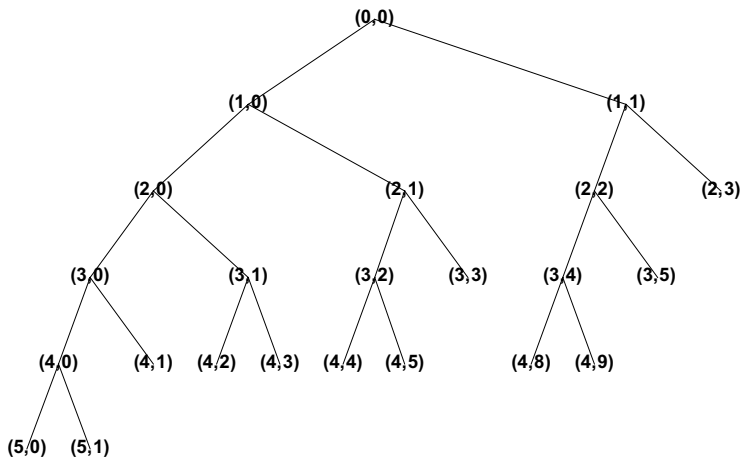

Fig.3 Best tree obtained using the "log energy" entropy type criterion.

China and Hunan provincial science and technology plan item (No.03GKY3115, 04FJ2003, 03JJY1010).

\section{REFERENCES}

[1] R. W. Liu, ed., Testing and Diagnosis of Analog Circuits and Systems, Van Nostrand Reinhold, USA, 1991.

[2] M. Catelani and A. Fort, "Soft Fault Detection And Isolation In Analog Circuits: Some Results And A Comparison Between A Fuzzy Approach And Radial Basis Function Networks", IEEE Trans Instrument Meas, vol.51(2), pp.196-202, Apr.2002.

[3] R.Spain and S.Upadhyaya, "Linear Circuit Fault Diagnosis Using Neuromorphic Analyzers", IEEE Trans Circuits Syst-II, vol.44(3), pp.188-196, Mar. 1997

[4]Y.G. He, X.J. Luo, and Q.G. Yuan, "A Neural-Based Nonlinear L1 Norm Optimization Algorithm for Diagnosis Of Networks", $J$. of Electronics, vol.15(4), pp.365-371, 1998

[5] W. Hochwald and J.D. Bastian, "A DC approach for analog fault dictionary determination", IEEE Trans. Circuits Syst, vol.CAS-26, pp.523-529, July 1997.

[6] M.Catelani and M.Gori, "On the application of neural network to fault diagnosis of electronic analog circuits", Measurement, vol.17, pp.73-80, 1996

[7] Y. He, Y. Ding and Y. Sun, "Fault diagnosis of analog circuits with tolerances using artificial neural networks," Proc. IEEE APCCAS, pp.292-295, Tianjin, 2000.

[8] Y. He and Y. Sun, "A neural-based L1-norm optimization approach for fault diagnosis of nonlinear circuits with tolerances," IEE Proc. Circuits, Devices and Systems, Vol.148, No.4: 223-228 2001.

[9] Y. He and Y. Sun, "Fault isolation in nonlinear analog circuits with tolerance using the neural-network based L1-norm," Proc. IEEE Int. Symp. on Circuits and Systems, pp.854-857, 2001.

[10] M. Aminian and F. Aminian, "Neural-network based analog circuit fault diagnosis using wavelet transform as preprocessor", IEEE Trans.Circuits Syst-II, vol.47(2), pp.151-156, 2000

[11] F. Aminian, M. Aminian and H.W. Collins, "Analog Fault Diagnosis of Actual Circuits Using Neural Networks", IEEE Trans. Instrum. Meas, Vol.51(3), pp544-550, June 2002.

[12] C. Hu, J. Zhang etc, Systematic Analysis and Design based on MATLAB-- Wavelet Analysis, China: Xi'an, 1999.

[13] M. R. Azimi-Sadjadi, D. Yao and Q. Huang, "Underwater Target Classification Using Wavelet Packets and Neural Networks", IEEE Trans Neural Net., Vol.11(3), pp:784-793, May 2000. 